Research Article

\title{
Study on Unsteady Cavitation Flow and Pressure Pulsation Characteristics in the Regulating Valve
}

\author{
Liu Xiumei $\mathbb{D}^{1,2}$ He Jie, ${ }^{1}$ Li Beibei $\mathbb{D},{ }^{1,2}$ Zhang Chi, ${ }^{1}$ Xu Huawen, ${ }^{1}$ Li Wei $\mathbb{D},{ }^{1,2}$ \\ and Xie Fangwei $\mathbb{D}^{1}$ \\ ${ }^{1}$ School of Mechatronic Engineering, China University of Mining and Technology, Xuzhou 221116, China \\ ${ }^{2}$ Center of Urban Emergency Technology and Equipment, China University of Mining and Technology, Xuzhou 221116, China
}

Correspondence should be addressed to Liu Xiumei; liuxiumei_lxm@126.com and Li Beibei; libeibeimail@163.com

Received 4 December 2020; Revised 30 December 2020; Accepted 6 January 2021; Published 16 January 2021

Academic Editor: Ling Zhou

Copyright (C) 2021 Liu Xiumei et al. This is an open access article distributed under the Creative Commons Attribution License, which permits unrestricted use, distribution, and reproduction in any medium, provided the original work is properly cited.

\begin{abstract}
A combined numerical-experiment investigation on the unsteady cavitation flow and pressure fluctuation characteristics in the regulating valves is conducted in this paper. The cavitation flow in the regulating valve is an unsteady and periodic flow which could be divided into fixed and travelling cavitation bubbles. The fixed cavitation bubbles are formed in the gap in the initial stage and then fell off and formed the travelling cavitation bubbles because of the re-entrant jet. The travelling cavitation bubbles move downstream, oscillate, and break up into several smaller bubbles. Changes in the length/radius ratio $\left(L / R_{0}\right)$ of the valve spool is an important factor affecting unsteady cavitation flow and pressure pulsation characteristics in the regulating valve. The length of travelling cavitation bubbles increases firstly and then decreases with increasing time. With the increase of the length/radius ratio $\left(L / R_{0}\right)$, the oscillation period of cavitation bubbles also increases. In the initial stage of cavitation bubbles, the velocity distribution inside the regulating valve is relatively stable, and no re-entrant jet could be found although $L / R_{0}$ is different. In the collapse stage of cavitation bubbles, the velocity distribution becomes extremely unstable because the collapsing cavitation bubbles affect the pressure drop and velocity field in the flow channel. Furthermore, the amplitude of pressure pulsation increases gradually, and the peak time of the pressure pulsation is gradually delayed while increasing the length/diameter ratio.
\end{abstract}

\section{Introduction}

The inner flow characteristic of the regulating valve has a direct influence on its performance. The cavitation inception, development, and collapse in the flow passage will lead to pressure oscillation in the regulating valve, vibration, noise, early valve failures, and affecting the stability of the hydraulic system [1-3]. Therefore, it is essential to understand thoroughly the complex flow characteristics inside the valve in order to improve the running safety of the regulating valve [4-6].

Unsteady cavitation flow and pressure pulsation characteristics in the regulating valve have been taken into account in more recent studies. In experiments, laser Doppler anemometry [7], particle image velocimetry [8], and high-speed camera-based method [9] were used to observe the cavitation phenomena occurring in the valve. Computational fluid dynamics (CFD) is expected to be another effective tool to solve the problem in fluid dynamics [10]. Ma et al. [11] presented the cavitation flow characteristics of the control valve using a Schnerr and Sauer cavitation model. The standard $k-\varepsilon$ turbulence model was selected for presenting the pressure and velocity distribution numerically [12]. Semrau et al. [13] confirmed that the cavitation bubble is an essential cause for noise generation. Ye et al. [14] deduced the relationship among the discharge coefficient, the groove geometry, flow condition, and fitting coefficients. Jin et al. [6] investigated cavitation flow in a sleeve regulating valve with different valve core shapes. Liang et al. [15] pointed out that vortex flow is the reason for the occurrence of the cavitation bubble. Yuan et al. [16] investigated the internal flow dynamics inside a poppet valve, specially made emphasis on cavitation-vortex interaction. Qiu et al. [17] discussed the pressure drop, velocity, and vapor volume distribution in the regulating valves, and they pointed out that the effects of the pressure difference on the cavitation intensity. Sayyaadi [18] found that higher cavitation numbers would induce the simpler fluctuation mode. Wang et al. [19] pointed that the 
re-entrant jet is the reason for choked cavitating flow and the cavitation regions could be divided into the inception and developing regions, fusion regions, and collapse regions in a venturi reactor. Qian et al. [20] discussed relationships between the valve core opening and the outlet flux under different valve core shapes and give a theoretical guide for the optimization of the valve core. There are many research that focus on cavitation flow in hydraulic valves in recent years, especially on the steady flow characteristics. The mechanism of cavitation flow in the regulating valve is still not clear, which are important to capture, study, and assess the risks of cavitation erosion. Thus, the unsteady cavitation flow and pressure pulsation characteristics in the regulating valve are discussed combining with high-speed photography and numerical simulation technology in our paper. The effect of the length/radius ratio $\left(L / R_{0}\right)$ of the regulating valve spool on the periodic cavitation flow and pressure fluctuation characteristics are also discussed.

\section{Numerical and Experimental Methods}

The commercial software ANSYS Fluent 15.0 is employed for numerical calculation of cavitation flow in the regulating valve.

2.1. Mathematical Modeling of Flow. The continuity equation can be written as follows:

$$
\frac{\partial}{\partial t}\left(\rho_{m}\right)+\nabla \cdot\left(\rho_{m} \vec{v}\right)=0 .
$$

The momentum equation can be written as follows:

$$
\frac{\partial}{\partial t}\left(\rho_{m} \vec{v}\right)+\nabla \cdot\left(\rho_{m} \vec{v} \vec{v}\right)=-\nabla p+\nabla\left[\mu_{m}\left(\nabla \vec{v}+\nabla^{T}\right)\right] .
$$

Here, $\mu_{m}$ is the viscosity of the mixture; $\rho_{m}$ is the mixture density defined as $\rho_{m}=\rho_{v} \alpha_{v}+\rho_{l}\left(1-\alpha_{v}\right) ; \rho_{v}$ and $\rho_{l}$ are the vapor density and the liquid density, respectively, and $\vec{v}$ is the mass-averaged velocity; $\alpha_{v}, 1-\alpha_{v}$ is the vapor volume fraction and liquid volume fraction, respectively; and $\nabla P$ is the gradient of pressure $P$.

The net mass transfer from liquid to vapor is often described by the Schnerr-Sauer cavitation model, which could be expressed as

$$
\begin{aligned}
& R_{e}=\frac{\rho_{l} \rho_{v}}{\rho_{m}} \alpha_{v}\left(1-\alpha_{v}\right) \frac{3}{R_{B}}\left(\frac{2}{3} \frac{P_{v}-P}{\rho_{l}}\right)^{1 / 2}, \quad P \leq P_{v}, \\
& R_{c}=\frac{\rho_{l} \rho_{v}}{\rho_{m}} \alpha_{v}\left(1-\alpha_{v}\right) \frac{3}{R_{B}}\left(\frac{2}{3} \frac{P-P_{v}}{\rho_{l}}\right)^{1 / 2}, \quad P \geq P_{v} .
\end{aligned}
$$

In the formula, $R_{B}$ is the bubble radius $R_{B}=\left(\left(\alpha_{v} / 1\right.\right.$ $\left.\left.-\alpha_{v}\right)(3 / 4 \pi)(1 / N)\right)^{(1 / 3)} ; P_{v}$ is the saturation vapor pressure; $n$ is the number of bubbles per volume of liquid, the model takes $n=10^{13}$.

Furthermore, mixture model, Schnerr-Sauer cavitation model, and multiphase flow model together with a modified RNG $\mathrm{k}-\varepsilon$ turbulence model are used to describe the evolution of unsteady cavitation flow inside the regulating valve. In order to improve the convergence and stability of the numerical simulations, the calculation result of steadystate cavitation flow is set as the initial calculation result in the calculation of unsteady flows. The simulation time step is set to $0.1 \mu \mathrm{s}$.

2.2. Geometrical Model of Test Valve. The schematic diagram of the regulating valve is shown in Figure 1, which mainly contains the valve stem, spool, and seat. The spool is used to adjust the flow rate, whose head shape is composed of line segments and parabolic curves. Considering that the geometric construction of the regulating valve is symmetrical, a rotational axisymmetric model for the cavitation simulation inside the regulating valve is used. The total length of the flow passage of the regulating valve is $40.0 \mathrm{~mm}$, while the length of the flow section located upstream is $20.0 \mathrm{~mm}$. The opening degree of the regulating valve is $1.0 \mathrm{~mm}$, and the inlet and outlet diameters of the flow channel are $4.5 \mathrm{~mm}$ and $3.0 \mathrm{~mm}$, respectively.

The whole flow field was discretized with a mixed grid, and the orifice of the regulating valve and flow section located downstream is handled in encrypted form. The pressure-inlet boundary condition of computations is commonly applied as an inlet boundary condition, while the inlet pressure is $4.0 \mathrm{MPa}$. The pressure-outlet boundary condition of computations is commonly applied as an outlet boundary condition, while the outlet pressure is $1.0 \mathrm{MPa}$. The axisymmetric boundary condition is applied to the center axis. In addition, other boundaries are adopted wall boundary conditions. In order to guarantee the precision of our simulation and save computation time, the grid independence analysis is carried out in our paper. The grid number of 20359, 48939, 79208, and 125611 are tested, respectively. While the number of grids reaches 79208 , the change of average pressure on the valve section is less than $0.5 \%$ in one bubble lifetime, which demonstrates that the results are grid independent. Hence, the amount of grid 79208 is used in our simulation, and the minimum grid size is $0.01 \mathrm{~mm} \times 0.01 \mathrm{~mm}$.

2.3. Experimental Setup. To obtain the unsteady cavitation flow in the regulating valve, the cavitation visualization experiment system was established based on the dynamic similarity principle. Figure 2 illustrates the hydraulic circuit used in our experiment, which has been reported in Ref [21]. Oil is supplied by a pump from a fuel tank. A pressure relief valve is used to regulate the operating pressure, and an accumulator is used to attenuate the pressure pulsation in the hydraulic system. The inlet and outlet pressure of the regulating valve is detected with pressure gages, and the flow rate of a fluid medium is measured by a flow rate meter. No. 46 antiwear hydraulic oil is used as a fluid medium in our experiment, and the temperature of the oil remains around $319 \mathrm{~K}$ during operation-based maintenance on a fin fan cooler. 


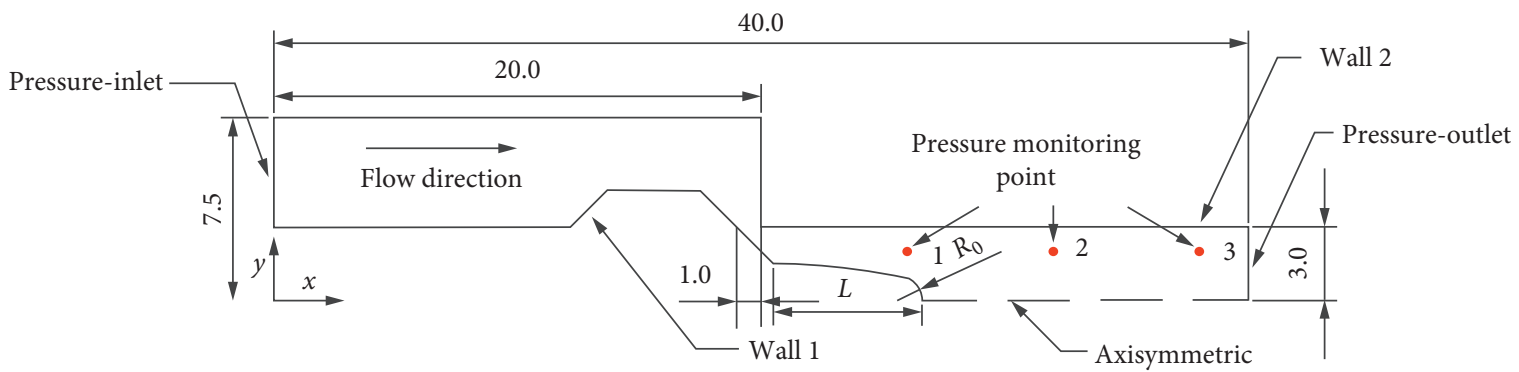

Figure 1: Numerical model of the regulating valve.

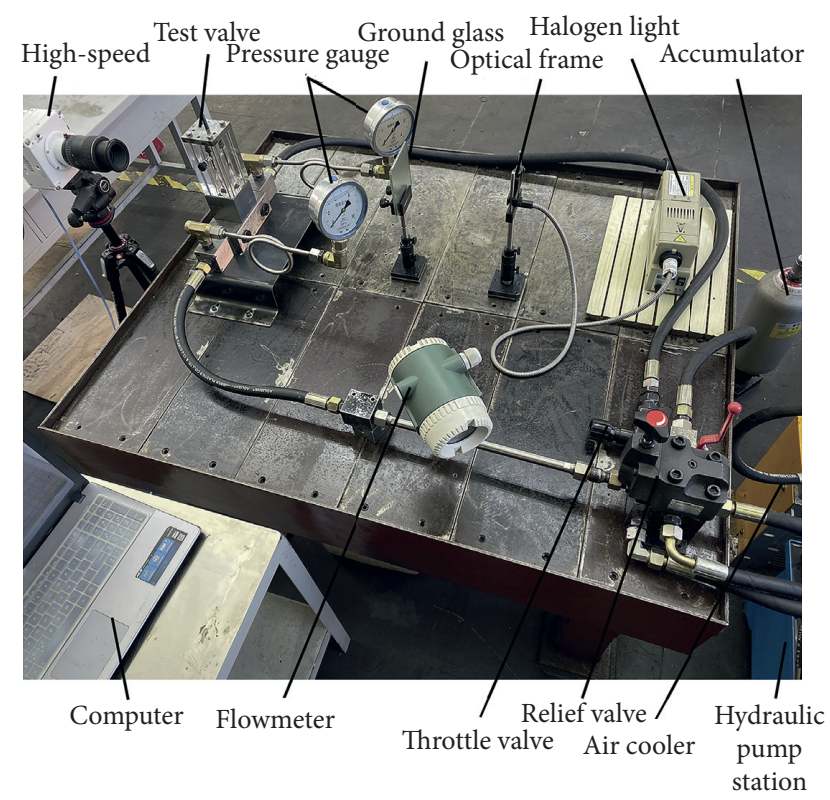

FIGURE 2: Illustration of experimental hydraulic circuit.

The valve house is made from polymethyl methacrylate (PMMA), which is used to observe the cavitation flow inside the channel. The size of transparent regulating valve is reduced to one-tenth of the actual geometry. A high-speed camera (PhantomVEO-710L) is used for consecutive image acquisition to investigate the evolution of cavitation bubbles. An optical-fibre source (LA-100USW) is used as an illuminate to improve image quality. The high-speed camera and the optical-fibre source are located on either side of the transparent valve. To catch the transient cavitation flow, the frame rate of 20,000 frames/second is selected during shooting.

\section{Results}

Figure 3 shows the evolution process of cavitation flow in the regulating valve with a pressure difference of $3.0 \mathrm{MPa}$, while an opening degree is at $1.0 \mathrm{~mm}$. The left column is numerical results while the right is corresponding experimental results. The results show that cavitation bubbles appear primarily in the gap between the valve seat and the valve core surface [6] because of sudden changes in the flow path induced by geometry of the flow domain such as sharp corners. Pressure drop mainly occurs in the narrow gap, resulting in the appearance of a cavitation bubble. This cavitation flow is an unsteady and periodic flow [19] in the regulating valve which could be divided into cavitation inception, development, detachment, and collapse. At the moment $t_{0}$, the flow pressure decreases due to decreasing the cross-sectional area of the orifice and fixed cavitation bubbles will be formed. During the moment $t_{0}$ to $t_{0}+0.30 \mathrm{~ms}$, these fixed cavitation bubbles develop downstream and its tail will fall off, forming travelling cavitation bubble as a result of re-entrant jets [19]. During the moment $t_{0}+0.30 \mathrm{~ms}$ to $t_{0}+0.60 \mathrm{~ms}$, the length of the fixed cavitation bubbles decreases gradually, while the travelling cavitation bubbles continue to develop and move downstream, whose width and length grow rapidly. With increasing time, this travelling cavitation bubbles reach their maximum shape and a re-entrant jet could be found upstream. At the moment $t_{0}+1.05 \mathrm{~ms}$, the travelling cavitation bubbles are split into many small-scale bubble column and collapse at their downstream end. At the moment $t_{0}+1.67 \mathrm{~ms}$, another reciprocation cycle of cavitation bubbles could be found at the orifice of the valve. Furthermore, the lifetime of the cavitation bubble obtained numerically is $1.67 \mathrm{~ms}$ while it is $1.60 \mathrm{~ms}$ obtained experimentally in Figure 3. The deviations of the bubble lifetime between the simulation and the experimental results are about $4 \%$. The difference between the numerical and experimental results may be caused by ignoring the changes in temperature and compressibility of the oil during the numerical calculation. Thus, the accuracy of the numerical model used in our paper is capable of predicting the evolution of cavitation bubbles in the regulating valve.

Figure 4 is the internal flow and the distribution of the cavitation bubbles at the moment $t_{0}+0.15 \mathrm{~ms}$ and $t_{0}+0.30 \mathrm{~ms}$, in order to analyze the phenomena in Figure 3. As shown in Figure 4, a constricted flow section locates in the valve throat because of the convergent-divergent structure. A high-velocity and low-pressure region appears behind the valve seat because of the sudden decreasing cross-section area $[6,17]$. The vapor phase volume fraction in the flow channel ranges from 0.25 to 0.95 , indicating that the cavitation observed are at higher intensities. Furthermore, Figure 4(a) shows that a recirculation flow with high speeds was generated between the tail of the fixed cavitation bubbles and the valve wall initially, whose flow direction is opposite to the main flow direction. When this recirculation flow meets the main flow, a re-entrant jet could be 

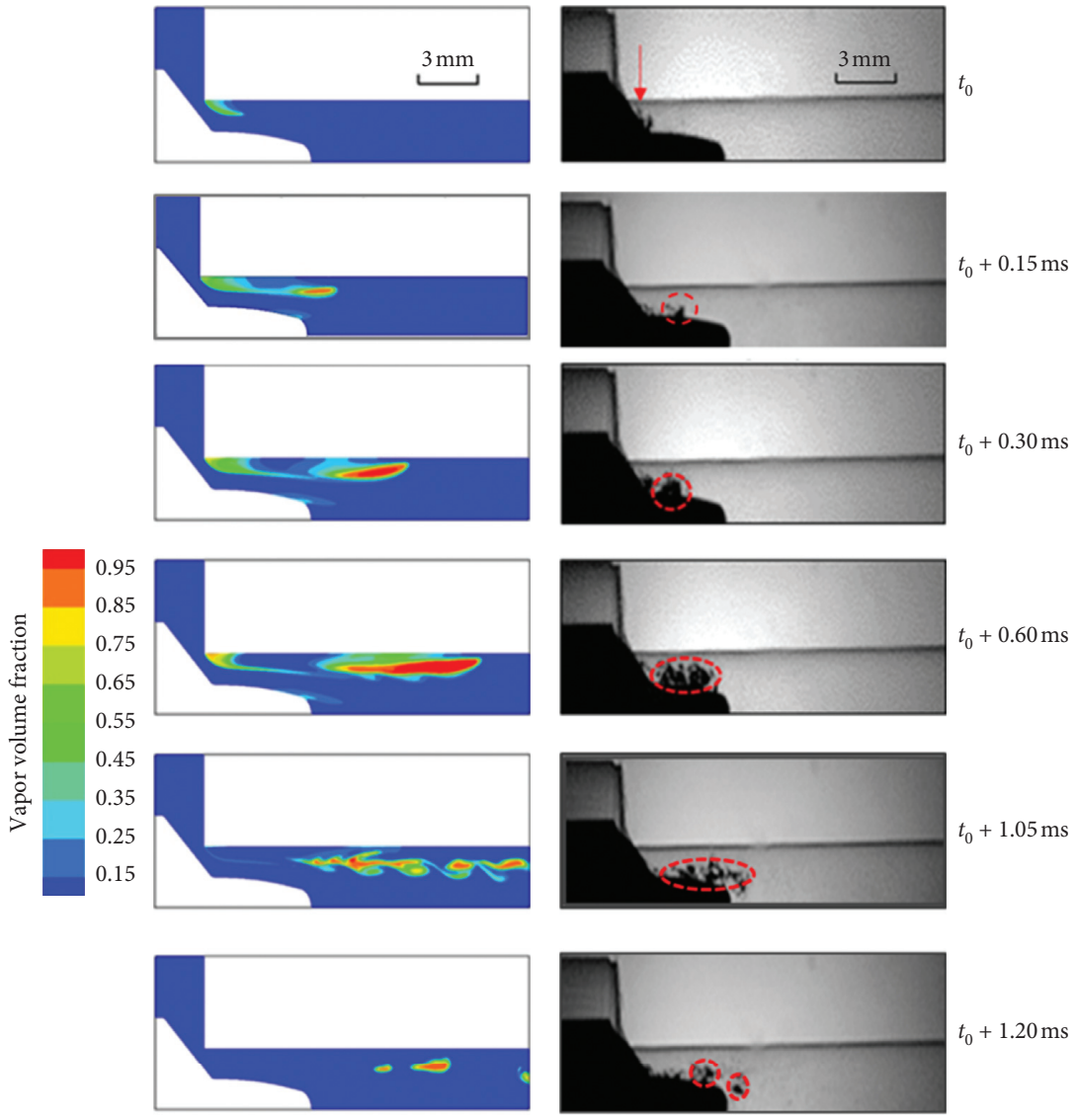

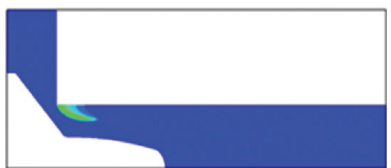

(a)

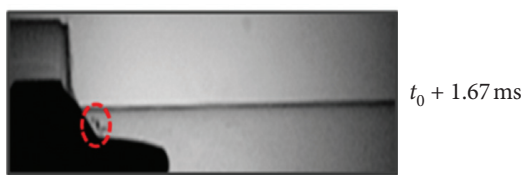

(b)

FIgURE 3: Typical evolution process of cavitation bubble.

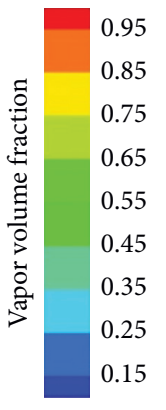

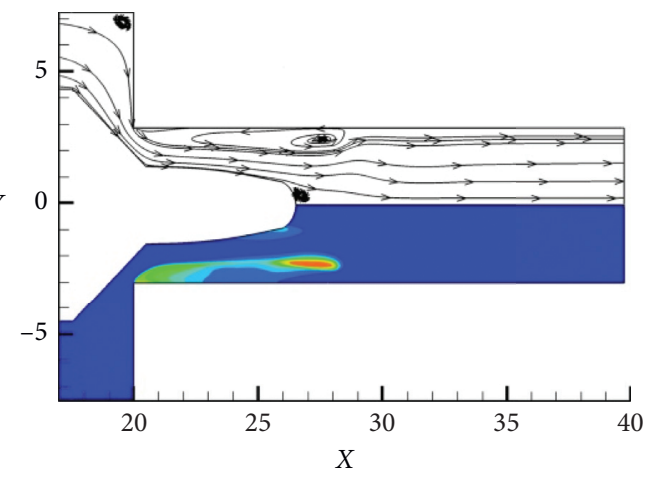

(a)

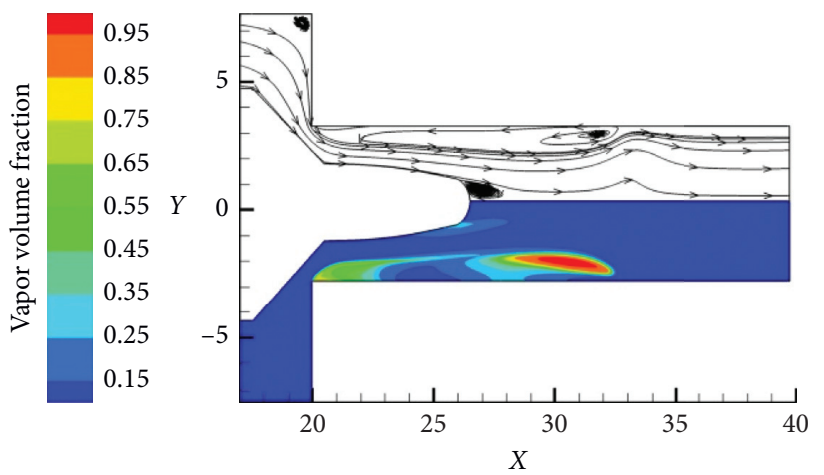

(b)

Figure 4: Flow charts (the upper part) and the distribution of vapour volume (the down part) at (a) $t_{0}+0.15 \mathrm{~ms}$ and (b) $t_{0}+0.30 \mathrm{~ms}$.

formed. This re-entrant jet will generate a large shear force, result in loss of fluid kinetic energy [15], and enhance the instability phenomenon of the cavitation bubble. Then, the fixed cavitation bubbles break into smaller travelling cavitation bubble and flowing downstream as shown in Figure 4(b). That is to say, the re-entrant jet is the main reason for cavitation breakup [22]. From the moment $t_{0}+0.15 \mathrm{~ms} t_{0}+0.30 \mathrm{~ms}$, the recirculation flow becomes long and narrow; the break-off 
phenomenon is more evident. So the area and the intensities of travelling bubbles increase with increasing time.

\section{Discussion}

4.1. Influence of the Length/Diameter Ratio on Unsteady Cavitation Flow. The flux characteristics of the regulated valve mainly depend on the valve spool shape [20]. Therefore, the characteristic parameter length/diameter ratio of the spool head is important in the optimized design to reduce the re-entrant jet velocity and increase the ability of antierosion. In order to evaluate the changes of cavitation flow in the regulating valve with different length/ diameter ratios of the valve core, the comparative analysis based on the numerical simulation is conducted, which are shown in Table 1 . Dimensionless parameters $\mathrm{L} / R_{0}$ is used to present the effect of the structural optimization design of the valve spool on the unsteady cavitation flow field, where $L$ is the length of the circular section and $R_{0}$ is the radius of the circular section of the valve spool head. Zhang et al. $[23,24]$ already reported that decreasing the radius of the spool head reduces the intensities of erosion-cavitation wear. So we only focus on the parameter the length of the circular section $L$.

Figure 5 is the variable length of travelling bubble verse time with different length/diameter ratios of the valve spool head. It could be found that the length of travelling cavitation bubble increases firstly and then decreases with different length/diameter ratios. As we discussed in Figure 3, the travelling bubble falls off and continues to grow downstream, whose width and length grow rapidly. With increasing time, this travelling cavitation bubble reaches its maximum shape and a re-entrant jet could be found upstream. Then, this travelling cavitation bubble collapses and is split into many small-scale bubble columns. Furthermore, when $L / R_{0}$ is 2.0 , the lifetime of the travelling cavitation bubble is about $0.6 \mathrm{~ms}$, and the maximum length of travelling cavitation bubble is about $9.1 \mathrm{~mm}$. When $\mathrm{L} / R_{0}$ rises up to 6.0 , the life time is $1.0 \mathrm{~ms}$, and the maximum length is $13.7 \mathrm{~mm}$. This is mainly due to the fact that the bigger the length/diameter ratio, more the time for the travelling cavitation bubble to grow along the channel. So the length of travelling bubble rises with the increasing length/diameter ratio.

As discussed in Figure 4, the cavitation bubble phenomenon could be found near the orifice of the regulating valve because of the shrinking flow area and increased flow velocity of the oil. When the bubble is at the collapse stage, the maximum velocity located near the gaps could be high to $100 \mathrm{~m} / \mathrm{s}$, as shown in Figure 6. This high-speed flow jet will result in the turbulent flow near the orifice. After passing through the throttle orifice, the flow of the fluid stabilizes again, and the velocity of the oil decreases gradually downstream. The characteristics of cavitation flow are closely related to the velocity field in the flow passage. Therefore, four points $a, b, c$, and $d$ are selected downstream at $29 \mathrm{~mm}, 32 \mathrm{~mm}, 35 \mathrm{~mm}$, and $38 \mathrm{~mm}$, as shown in Figure 6, in order to better explain the change of the velocity field in the flow channel.
TABLE 1: The length/diameter ratio of valve spool.

\begin{tabular}{lccccc}
\hline Parameters & 1 & 2 & 3 & 4 & 5 \\
\hline$L(\mathrm{~mm})$ & 2.0 & 3.0 & 4.0 & 5.0 & 6.0 \\
$R_{O}(\mathrm{~mm})$ & 1.0 & 1.0 & 1.0 & 1.0 & 1.0 \\
$L / R_{0}$ & 2.0 & 3.0 & 4.0 & 5.0 & 6.0 \\
\hline
\end{tabular}

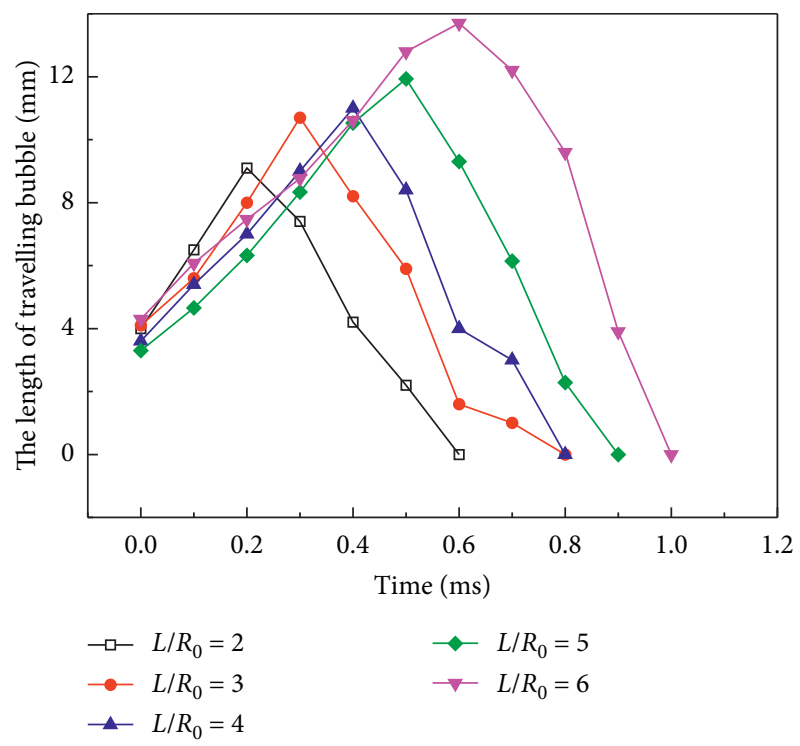

FIGURE 5: The variable in length of travelling cavitation bubble with different length/diameter ratios.

Figure 7 shows the distribution of the axial velocity downstream in the four selected radial cross sections, $x=29 \mathrm{~mm}, 32 \mathrm{~mm}, 35 \mathrm{~mm}$, and $38 \mathrm{~mm}$, at the moment of cavitation inception $\left(T_{0}\right)$ with different length/diameter ratios. The evolution of the axial velocity at the same radial cross section with different length/diameter ratios is almost the same. Because the fixed cavitation bubble is just occurred at the moment of cavitation inception $T_{0}$, the flow field downstream is relatively stable, and there is no re-entrant jet flow. And the flow velocity of the oil near the wall of the valve is lower than in the center of the flow channel. At $29 \mathrm{~mm}$, the maximum velocity in the lower half of the channel is higher than it in the center, because of a high-speed flow jet which originates from the narrow gap. With the increasing flow distance, as shown from (a) to (d), the maximum axial velocity decreases gradually, which is mainly due to the effect of the viscous resistance of the oil which reduces the maximum velocity and shrinks the high-speed flow area. Furthermore, the maximum of the axial speed, at $x=29 \mathrm{~mm}$, $32 \mathrm{~mm}, 35 \mathrm{~mm}$, and $38 \mathrm{~mm}$, is decreasing with increasing the length/diameter ratio. A larger length/diameter ratio lengthens the throttle effect enlargers. And the flow resistance in the channel will increase, which will induce the decreasing maximum axial velocity.

As we know, the collapse stage is important to the cavitation flow in the regulating valve. Figure 8 is the corresponding distribution of the axial velocity in the four different selected points at the cavitation collapsing time (3T/4, Tis the collapse time). The flow velocity of the oil near 


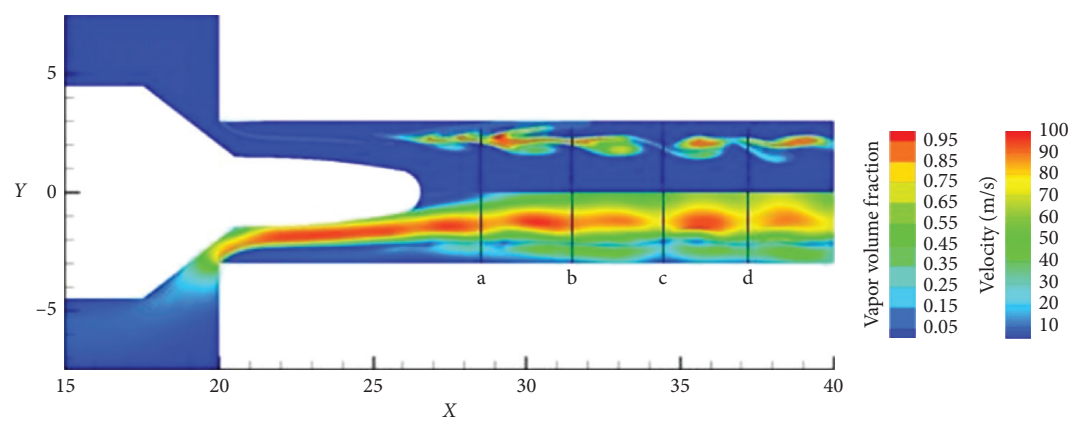

FIGURE 6: The distribution of a gas volume fraction (upper part) and velocity (down part) at the cavitation collapse process.

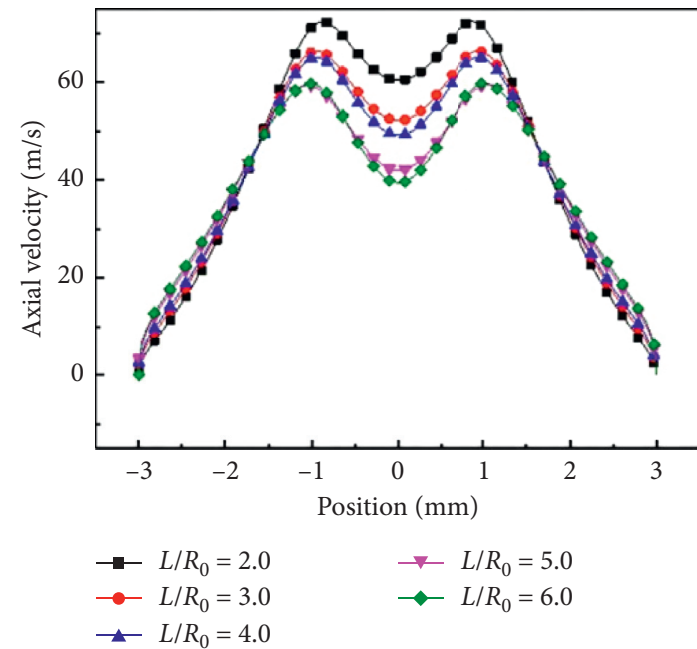

(a)

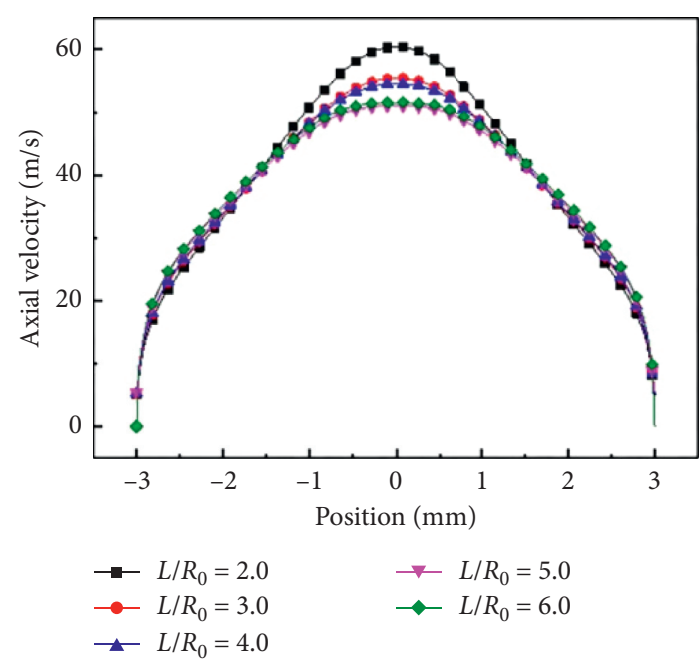

(c)

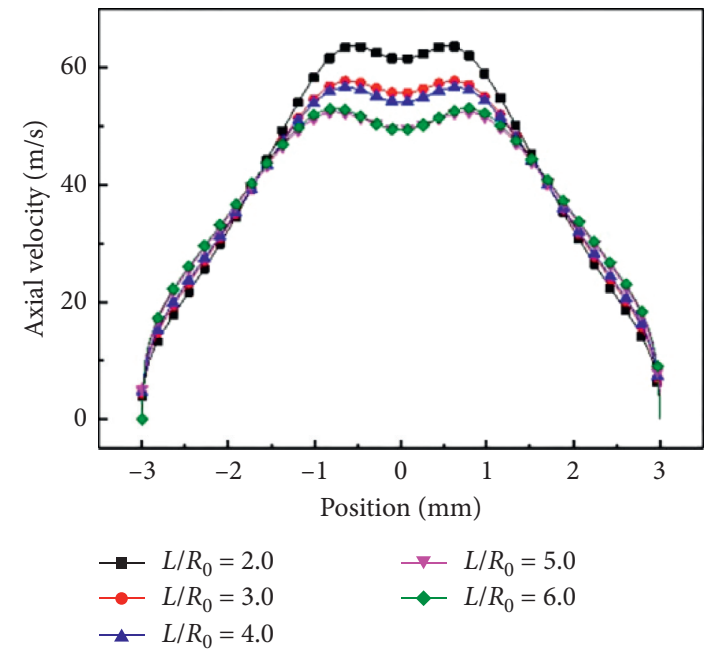

(b)

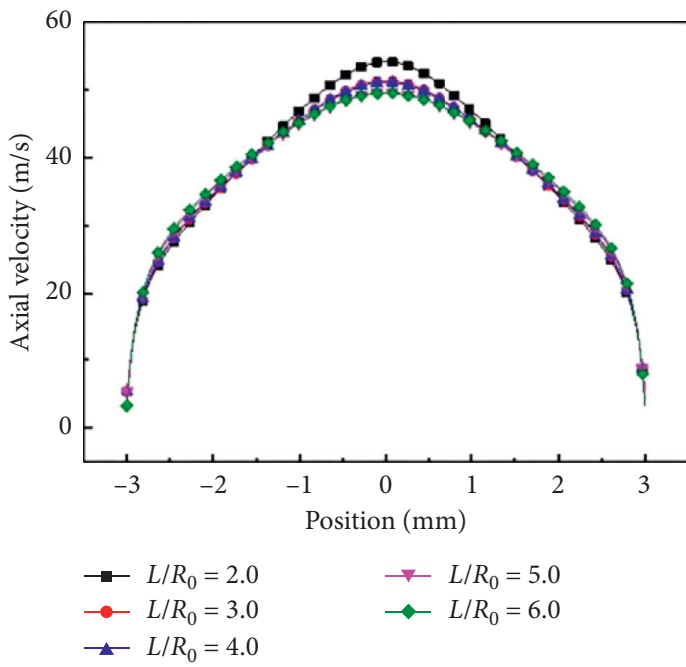

(d)

Figure 7: The distribution of the axial velocity downstream in $x=29 \mathrm{~mm}, 32 \mathrm{~mm}, 35 \mathrm{~mm}$, and $38 \mathrm{~mm}$ at the moment of cavitation inception (T. $T_{0}$. (a) $x=29 \mathrm{~mm}$. (b) $x=32 \mathrm{~mm}$. (c) $x=35 \mathrm{~mm}$. (d) $x=38 \mathrm{~mm}$.

the wall of the valve is lower than in the center of the flow channel and a re-entrant jet flow could be found at $1 \mathrm{~mm}$ away from the wall. This re-entrant jet flow reduces the fluid velocity near the wall. And the re-entrant jet flow could always be found downstream at the moment 3T/4 with different length/diameter ratios, whose intensity and position are different. Different characteristics of the re-entrant jet and the turbulence flow in the channel result in complex velocity distributions with different length/diameter ratios. At the moment $3 T / 4$, the cavitation flow is in the final stage 


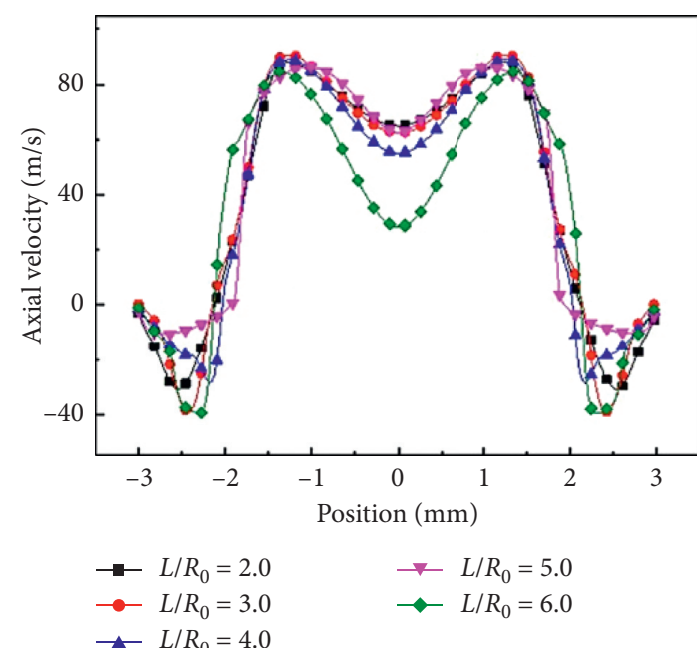

(a)

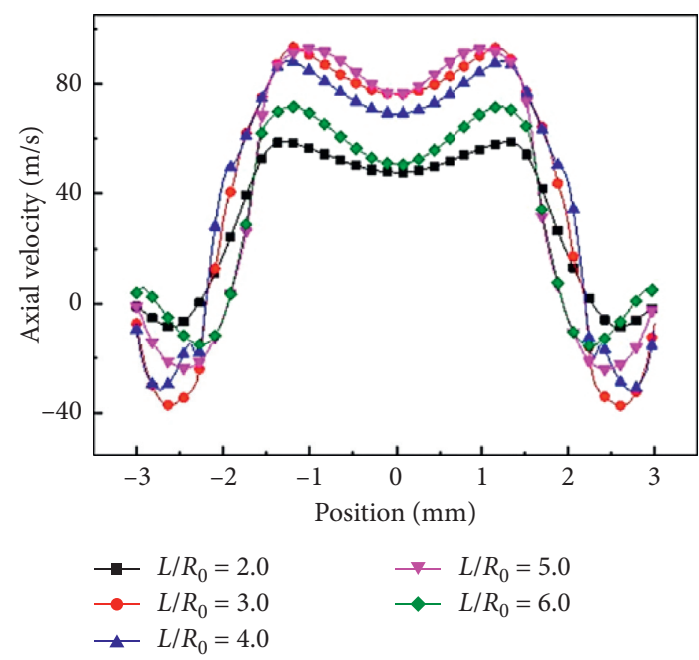

(c)

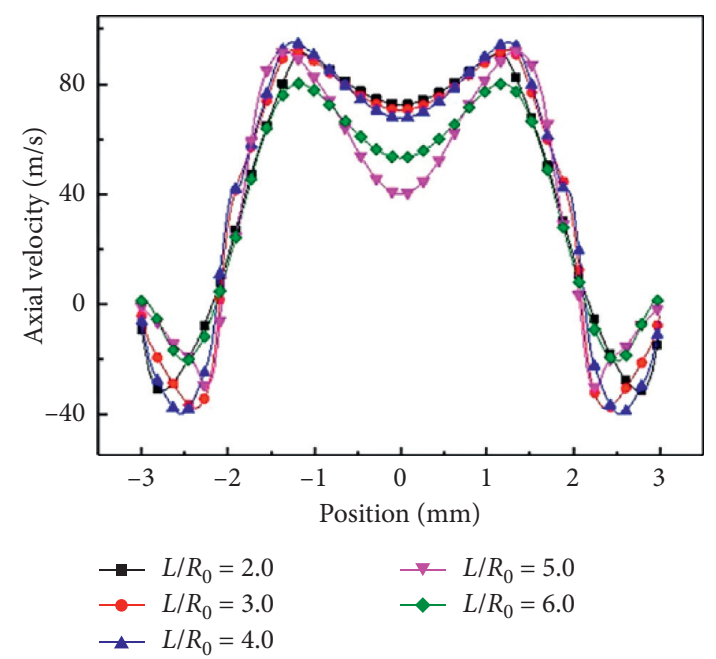

(b)

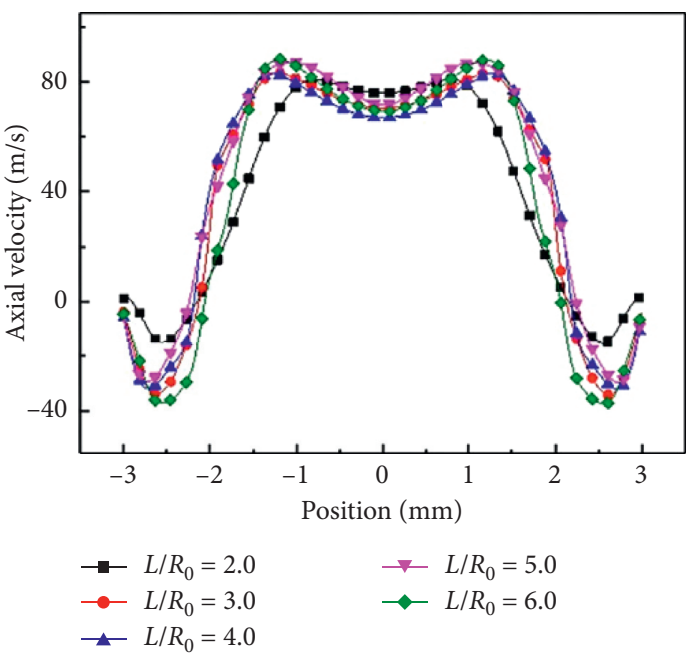

(d)

Figure 8: The distribution of the axial velocity downstream at $x=29 \mathrm{~mm}, 32 \mathrm{~mm}, 35 \mathrm{~mm}$, and $38 \mathrm{~mm}$ at the moment of $3 \mathrm{~T} / 4$. (a) $x=29 \mathrm{~mm}$. (b) $x=32 \mathrm{~mm}$. (c) $x=35 \mathrm{~mm}$. (d) $x=38 \mathrm{~mm}$.

of the collapse of a cavitation bubble; the travelling cavitation bubbles are split into many small-scale bubble columns at $3 T / 4$. When these small travelling cavitation bubble clouds enter into in area at the higher pressure, they will suddenly collapse resulting in unstable velocity distribution downstream. Because the collapse of small travelling cavitation bubbles and the rebirth of cavitation bubbles occur alternately at the different positions of the flow channel, there was no significant change in the value and location of the maximum velocity.

The re-entrant jet is the key factor leading to serious cavitation corrosion and failure in the head of the valve core [24]. Figure 9 shows the axial velocity distribution along the centerline of the valve spool, which shows that a re-entrant jet could be found at the head of the valve spool, and the size and length of this re-entrant jet also increase with the increasing length/diameter ratio. While the $L / R_{0}$ increases from 2.0 to 6.0 , the maximum axial velocity decreases. The reason is that the existence of the re-entrant jet, whose direction is opposite to the mainstream, consumes energy. In addition, the effect of the increasing length/diameter ratio is similar to two orifices in series, which means increasing total liquid resistance, and reduces the maximum axial velocity. By decreasing the length/diameter ratio, the re-entrant jet velocity and cavitation area on the parabolic surface could be decreased significantly. Therefore, the intensities of cavitation erosion could be mitigated. Reducing the length/radius ratio of the valve spool could weaken the re-entrant jet, weakening the damage of cavitation erosion and extending the service life of the regulating valve.

4.2. Influence of the Length/Diameter Ratio on Pressure Pulsation. In order to obtain the influence of unsteady cavitation flow on pressure pulsation, three monitoring points 1, 2, and 3 at the downstream of the channel are selected to monitor the pressure pulsation, marked by red dots in Figure 1. As we discussed above, the collapse of the 


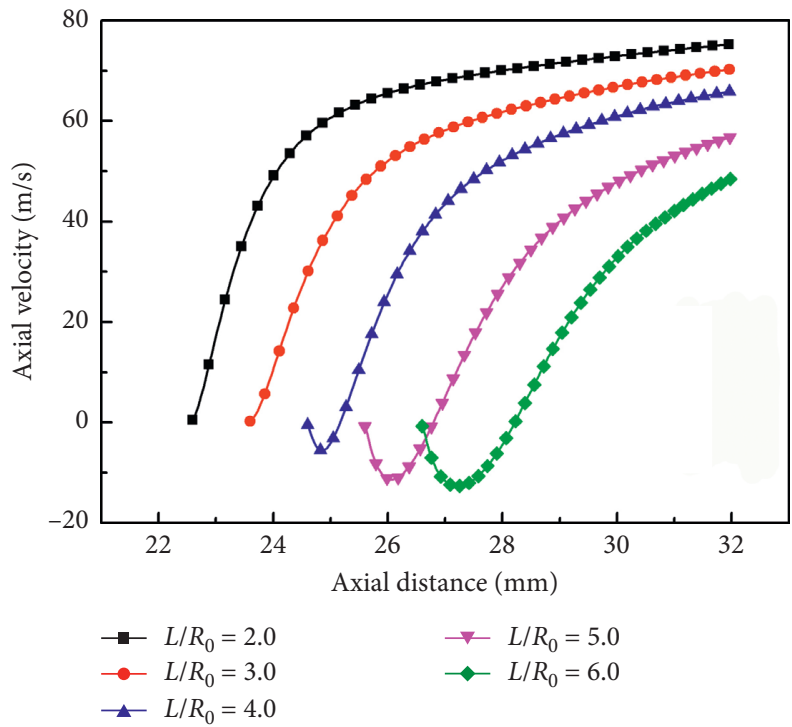

FIgURE 9: The axial velocity distribution along the centerline of the valve spool with different length/diameter ratios.

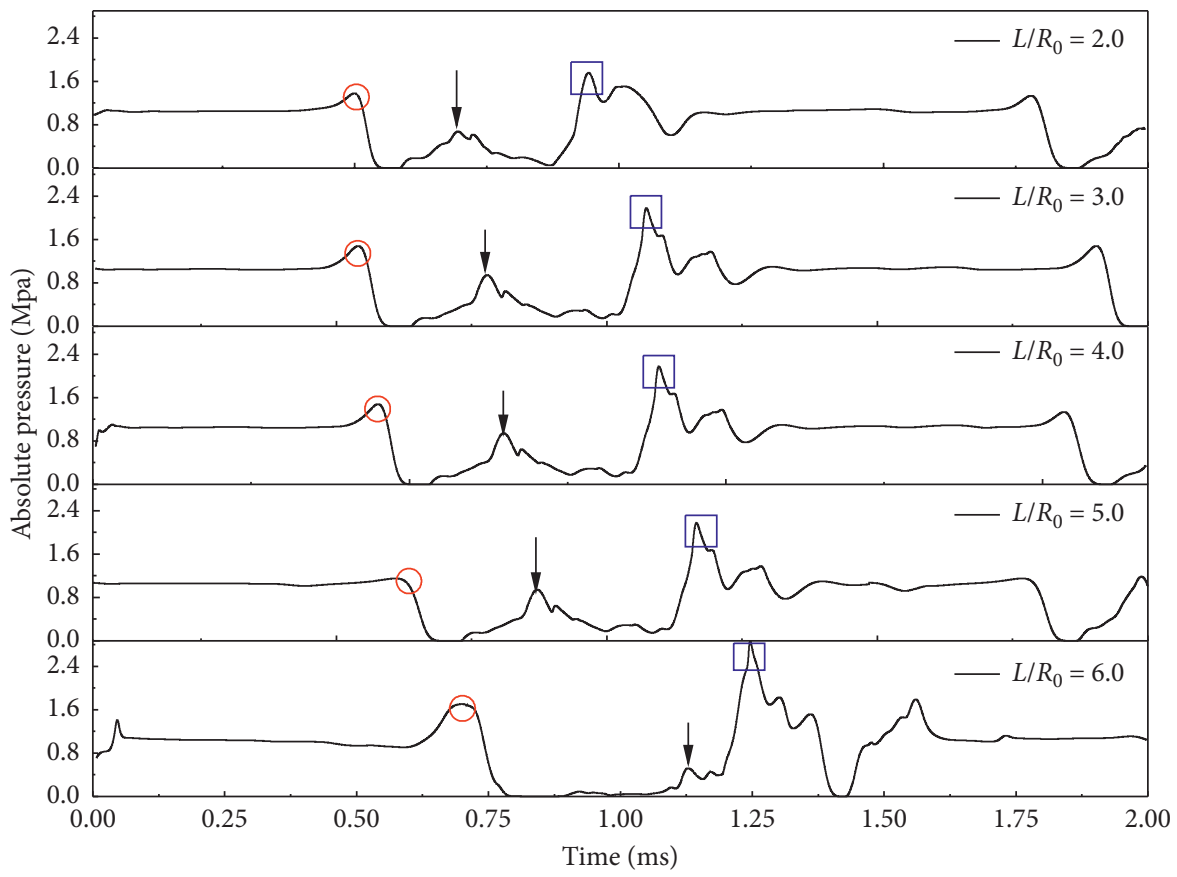

FIgURE 10: Pressure pulsation at the monitoring point 3 .

travelling bubbles is mainly found at its downstream end and the pressure pulsations caused by the collapse of the travelling bubbles are monitored at the typical monitor point 3 , as shown in Figure 10. When $L / R_{0}$ is 2.0 , the absolute pressure at point 3 decreases rapidly around $0.5 \mathrm{~ms}$ because the monitoring point 3 is covered by the travelling cavitation bubbles downstream. A trough of wave could be found, which follows by a circular area in Figure 10, and its absolute pressure is lower than the saturated steam pressure of the oil. Then, the collapse of the travelling cavitation bubbles happens and a peak of the wave will appears as marked by a square area in Figure 10, whose value is about 1.8 MPa. Later, a new life cycle of the cavitation bubble starts again around $2 \mathrm{~ms}$, and the absolute pressure at the point 3 recovers to the outlet pressure. It is worth noting that there will be a partial low-pressure pulsation, pointed by a black arrow in Figure 10, which is caused by the detachment of travelling bubbles from the fixed cavitation bubble. Furthermore, while the length/ diameter ratio increases from 2.0 to 6.0 , the amplitude of the peak of the wave increases gradually and the peak time of the pressure pulsation is gradually delayed. More cavitation bubbles fell off and collapse at a larger length/diameter ratio, 
that is, the reason why the amplitude of pressure pulsation becomes larger. In addition, the trough of the wave also delays at a larger length/diameter ratio; this is mainly due to severe cavitation that occurs and exerts its blocking effects on fluid flow, and the flow speed of travelling cavitation bubble towards downstream decrease. So the time travelling cavitation bubble reaches the monitoring 3 becomes longer.

\section{Conclusion}

The influence of the length/diameter ratio of the valve spool head on unsteady cavitation flow characteristics in the hydraulic regulating valve is analyzed experimentally and numerically. The cavitation flow in the regulating valve could be divided into fixed and travelling cavitation bubbles, which is an unsteady and periodic flow. The cavitation lifetime generally increases firstly and then decreases with increasing time at different length/diameter ratios of the valve spool head. In the initial cavitation stage, the velocity field distribution in the regulating valve is stable and there is no re-entrant jet even the length/diameter ratio of the valve spool head is different. In the cavitation collapsing stage, unstable velocity field distribution could be found in the flow passage of the regulating valve. Furthermore, decreasing the length/diameter ratio of the valve spool is an effective method to suppress the intensity of the re-entrant jet and thus relieves cavitation erosion of the valve spool. Cavitation bubble occurs less intensely, and less cavitation bubbles fall off and split into many small-scale bubbles with smaller length/diameter ratio, so the amplitude of pressure pulsation shrinks. As different length/diameter ratios of the valve spool is applicable to different operating conditions, we cannot simply judge which valve spool shape is optimal.

\section{Data Availability}

The data used to support the findings of this study are available from the corresponding author upon request.

\section{Conflicts of Interest}

The authors declare that they have no conflicts of interest.

\section{Acknowledgments}

This work was supported by the National Natural Science Foundation of China (Grant nos. 51875559 and U1810123), the Fundamental Research Funds for the Central Universities (2015XKMS026), and the Priority Academic Program Development of Jiangsu Higher Education Institutions (PAPD).

\section{References}

[1] B. Charrière, J. Decaix, and E. Goncalvès, "A comparative study of cavitation models in a venturi flow," European Journal of Mechanics - B/Fluids, vol. 49, pp. 287-297, 2015.

[2] A. Aghajani, "Controlling cavitation damage in pumps on oil offshore platforms," Materials Performance, vol. 55, pp. 52-54, 2016.
[3] L. Bai, L. Zhou, X. Jiang, Q. Pang, and D. Ye, "Vibration in a multistage centrifugal pump under varied conditions," Shock and Vibration, vol. 2019, Article ID 2057031, 9 pages, 2019.

[4] H. Wang, H. Xu, Y. Zhang, S. Chen, Z. Zhao, and J. Chen, "Design of a bio-inspired anti-erosion structure for a water hydraulic valve core: an experimental study," Biomimetics, vol. 4, no. 3, 63 pages, 2019.

[5] X. Wang, H. Liu, and G. Man, "Simulation and experimental study on cavitation and noise of hydraulic cone valve," Industrial Lubrication and Tribology, vol. 71, no. 2, pp. 318-323, 2019.

[6] Z.-J. Jin, C. Qiu, C.-H. Jiang, J.-Y. Wu, and J.-Y. Qian, "Effect of valve core shapes on cavitation flow through a sleeve regulating valve," Journal of Zhejiang University-Science A, vol. 21, no. 1, pp. 1-14, 2020.

[7] A. Sou, B. Biçer, and A. Tomiyama, "Numerical simulation of incipient cavitation flow in a nozzle of fuel injector," Computers \& Fluids, vol. 103, pp. 42-48, 2014.

[8] W. Shen, J. Zhang, Y. Sun, D.-J. Zhang, and J.-H. Jiang, "Effect of cavitation bubble collapse on hydraulic oil temperature," Journal of Central South University, vol. 23, no. 7, pp. 16571668, 2016.

[9] J. Jablonská, M. Kozubková, D. Himr, and M. Weisz, "Methods of experimental investigation of cavitation in a convergent - divergent nozzle of rectangular cross section," Measurement Science Review, vol. 16, no. 4, pp. 197-204, 2016.

[10] G. J. Peng, X. Huang, L. Zhou, G. X. Zhou, and H. Zhou, "Solid-liquid two-phase flow and wear analysis in a large-scale centrifugal slurry pump," Engineering Failure Analysis, vol. 114, p. 11, 2020.

[11] H. Ma, T. Zhang, Q. An, Y. Tao, and Y. Xu, "Visualization experiment and numerical analysis of cavitation flow characteristics in diesel fuel injector control valve with different structure design," Journal of Thermal Science, vol. 27, no. 1, pp. 89-94, 2020.

[12] X.-J. Wang, W.-J. Li, C.-H. Li, and Y.-W. Peng, "Structure optimization and flow field simulation of plate type high speed on-off valve," Journal of Central South University, vol. 27, no. 5, pp. 1557-1571, 2020.

[13] S. Semrau, R. Skoda, W. Wustmann, and K. Habr, "Experimental and numerical investigation of noise generation due to acoustic resonance in a cavitating valve," Journal of Sound and Vibration, vol. 463, p. 21, 2019.

[14] Y. Ye, C.-B. Yin, X.-D. Li, W.-J. Zhou, and F.-F. Yuan, "Effects of groove shape of notch on the flow characteristics of spool valve," Energy Conversion and Management, vol. 86, pp. 1091-1101, 2014.

[15] J. Liang, X. Luo, Y. Liu, X. Li, and T. Shi, "A numerical investigation in effects of inlet pressure fluctuations on the flow and cavitation characteristics inside water hydraulic poppet valves," International Journal of Heat and Mass Transfer, vol. 103, pp. 684-700, 2016.

[16] C. Yuan, J. Song, L. Zhu, and M. Liu, "Numerical investigation on cavitating jet inside a poppet valve with special emphasis on cavitation-vortex interaction," International Journal of Heat and Mass Transfer, vol. 141, pp. 1009-1024, 2019.

[17] C. Qiu, C. H. Jiang, H. Zhang, J. Y. Wu, and Z. J. Jin, "Pressure drop and cavitation analysis on sleeve regulating valve," Processes, vol. 7, p. 16, 2019.

[18] H. Sayyaadi, "Instability of the cavitating flow in a venturi reactor," Fluid Dynamics Research, vol. 42, Article ID 055503, 2010.

[19] J. Wang, L. Wang, S. Xu, B. Ji, and X. Long, "Experimental investigation on the cavitation performance in a venturi 
reactor with special emphasis on the choking flow," Experimental Thermal and Fluid Science, vol. 106, pp. 215-225, 2019.

[20] J.-Y. Qian, C.-W. Hou, J. Mu, Z.-X. Gao, and Z.-J. Jin, "Valve core shapes analysis on flux through control valves in nuclear power plants," Nuclear Engineering and Technology, vol. 52, no. 10, pp. 2173-2182, 2020.

[21] X. Liu, Z. Wu, B. Li et al., "Influence of inlet pressure on cavitation characteristics in regulating valve," Engineering Applications of Computational Fluid Mechanics, vol. 14, no. 1, pp. 299-310, 2020.

[22] L. Lu, S. Xie, Y. Yin, and S. Ryu, "Experimental and numerical analysis on the surge instability characteristics of the vortex flow produced large vapor cavity in u-shape notch spool valve," International Journal of Heat and Mass Transfer, vol. 146, 2020.

[23] Z. Zheng, G. Ou, H. Ye et al., "Investigation on failure process and structural optimization of a high pressure letdown valve," Engineering Failure Analysis, vol. 66, pp. 223-239, 2016.

[24] Z. He, Y. Chen, X. Leng, Q. Wang, and G. Guo, "Experimental visualization and LES investigations on cloud cavitation shedding in a rectangular nozzle orifice," International Communications in Heat and Mass Transfer, vol. 76, pp. 108-116, 2016. 